\title{
LINEAR AND NON-LINEAR REGRESSION ANALYSIS FOR THE BIOSORPTION KINETICS OF METHYLENE BLUE
}

\author{
LUBOŠ VRTOCH, JOZEF AUGUSTÍN
}

\author{
Department of Biotechnology, University of SS Cyril and Methodius, J. Herdu 2, \\ Trnava, SK-917 01, Slovak Republic (vrtochl@ucm.sk)
}

\begin{abstract}
The nonviable biomass of Rhizopus sp. R-18, Penicillium candidum and Penicillium chrysogenum was studied for biosorption of methylene blue (MB). The sorption of MB was studied be performing batch kinetic experiments. Kinetic measurements showed that sorption of $\mathrm{MB}$ reached equilibrium in 4 hours. The batch sorption models, based on a pseudo-first, pseudo-second and pseudo- $n$th order were applied to predict the rate constant of sorption and the equilibrium capacity. The linear and nonlinear least-square methods were used to obtain the kinetic parameters. The best-fit model was identified using statistic analysis. The results showed that both linear and nonlinear form of pseudo- second order expression could be used to fit the experimental data but nonlinear method may be a better way to obtain the desired parameters. As well the pseudo n-th order kinetic model was successfully applied to the kinetic data. The order $(n)$ of adsorption reaction was found for all employed biosorbents: for Rhizopus sp. R-18 it had value $3.1, P$. candidum 3.0 and $P$. chrysogenum 3.8 .
\end{abstract}

Key words: biosorption, kinetics, methylene blue, regression analysis, fungi

\section{Introduction}

Environmental pollution due to technological development is one of the most important problems than men have to face. Biosorption as a separation process has aroused considerable interest during recent years. In wastewater treatment, biosorption is regarded as one of the most potent techniques for the removal of dyes (AKSU, 2005). Two important physico-chemical aspects for evaluation of a sorption process as a unit operation are the equilibrium of the sorption and the sorption kinetics (HO et al., 2000). The nature of sorption process will depend on physical or chemical characteristics of the adsorbent systems and also on the system conditions at which sorption processes may include ion exchange, chelation, physical and chemical sorption. Predicting the rate at which the pollutants removal takes place in a given solid/solution system is one of the most crucial factors for the effective sorption system design. Chemical kinetics explains how fast the rate of chemical reaction occurs and also the factors affecting the reaction rate. Several researchers have used different kinetic models to predict the mechanism involved in the sorption process. These models can be divided into two main types: diffusion based models and reaction based models (AL-DEGS et al., 2006; SVILOVIĆ and STIPIŠIĆ, 2009). In the present study, we used reaction based models, namely Lagergren pseudo-first order kinetic model, pseudo-second order model and pseudo- $n$th order kinetic model for prediction of batch sorption kinetics.

In the present study, a comparison between linear and non-linear regression method has been made to predict the best sorption kinetics and also to obtain the kinetic parameters. 


\section{Materials and methods}

\subsection{Biosorbents and absorbate}

Three different fungi were used as biosorbents, Rhizopus sp. R-18, Penicillium candidum and Penicillium chrysogenum. The Rhizopus sp. R-18 and P. candidum were obtained from the collection of microorganisms, SS. Cyril and Methodius University, Trnava, Slovakia. P. chrysogenum biomass in paste form was obtained from local antibiotics producing plant. The Rhizopus and P. candidum biomass was obtained by cultivation in the Czapek-Dox broth. After 7 days the biomass was harvested, washed with distilled water and dried at $60^{\circ} \mathrm{C}$ for $24 \mathrm{~h}$. Subsequently, dried biomass was powdered by using mortar and pestle. The powder was sieved to obtain the particle size fraction below $0.31 \mathrm{~mm}$. In a similar manner was utilized $P$. chrysogenum biomass, too.

The absorbate used in all the experiments was methylene blue (Basic Blue 9), a cationic dye. The stock solution of MB was prepared by dissolving $0.5 \mathrm{~g}$ of substance in one liter of distilled water. All working solutions of desired concentrations were prepared by diluting the stock solution with distilled water.

\subsection{Sorption kinetic experiments}

Kinetic experiments were conducted on the rotary shaker with constant agitation speed of $200 \mathrm{rpm}$, using $50 \mathrm{ml}$ Erlenmeyer flasks containing $10 \mathrm{ml} \mathrm{MB}$ solutions 200 $\mathrm{mg} . \mathrm{L}^{-1}, \mathrm{pH} 6.0$ and $0.03 \mathrm{~g}$ of each type of biosorbent for $360 \mathrm{~min}$. Samples were drawn from the mixture at pre-determined time intervals for analysis. The dye concentration in supernatants (3 $000 \mathrm{RPM}$ ) were estimated spectrophotometrically at $665 \mathrm{~nm}$.

\subsection{Regression analysis, goodness-of-fit measure and model comparison}

The kinetic parameters were evaluated by linear and/or non-linear regression analysis by using QC.Expert ${ }^{\circledR} 3.1$ and OriginPro ${ }^{\circledR}$ 7.0. In the present study, several error analysis methods were used in order to confirm the best-fitting kinetic model, namely the coefficient of determination $\left(R^{2}\right)$, the sum of the squares of the errors $(S S E)$, the sum of the absolute errors $(S A E)$ and Chi-square analysis (reduced $\left.\chi^{2}\right)$. We used one statistical approach for comparing models: Akaike's information criterion $(A I C)$.

\section{Results and discussion}

\subsection{Linear regression analysis}

A commonly used model for describing sorption kinetic is the pseudo-second order kinetic model. Because this kinetic model is non-linear, fitting this model to measured data requires a "trial and error" approach. Alternatively, a linearized version of this model (- at least four different version exist -) can be used (Table 1). A limitation to 
this approach, however, is that the transformation of data required for linearization can result in modifications of error structure, introduction of error into the independent variable, and alteration of the weight placed on each data point, often leading to difference in fitted parameter values between linear and non-linear version of the pseudo-second order kinetic model (KUMAR, 2006; BOLSTER and HORNBERGER, 2007). Table 2 shows kinetic parameters obtained by using linear equations of the four pseudo-second order kinetic models.

Table 1. Pseudo second-order kinetics and their linear forms, $Q_{t}, Q_{e}$-the amount of dye adsorbed at any time $t$ and at equilibrium, respectively; $k_{2}$-the rate constant of sorption (HO, 2006).

\begin{tabular}{|c|c|c|c|}
\hline Kinetic model & Linear form & Plot & Parameters \\
\hline Model 1 & $\frac{t}{Q_{t}}=\frac{1}{k_{2} Q_{e}^{2}}+\frac{1}{Q_{e}} t$ & $t / Q_{t}=f(t)$ & $\begin{array}{c}Q_{e}=1 / \text { slope } \\
k_{2}=\text { slope }^{2} / \text { intercept } \\
h=1 / \text { intercept }\end{array}$ \\
\hline Model 2 & $\frac{1}{Q_{t}}=\left(\frac{1}{k_{2} Q_{e}^{2}}\right) \frac{1}{t}+\frac{1}{Q_{e}}$ & $1 / Q_{t}=f(1 / t)$ & $\begin{array}{c}Q_{e}=1 / \text { intercept } \\
k_{2}=\text { intercept }^{2} / \text { slope } \\
h=1 / \text { slope }\end{array}$ \\
\hline Model 3 & $Q_{t}=Q_{e}-\left(\frac{1}{k_{2} Q_{e}}\right) \frac{Q_{t}}{t}$ & $Q_{t}=f\left(Q_{t} / t\right)$ & $\begin{array}{c}Q_{e}=\text { intercept } \\
k_{2}=-1 /\left(\text { slope } *_{\text {intercept })}\right. \\
h=- \text { intercept } / \text { slope }\end{array}$ \\
\hline Model 4 & $\frac{Q_{t}}{t}=k_{2} Q_{e}^{2}-\frac{k_{2} Q_{e}^{2} Q_{t}}{Q_{e}}$ & $Q_{t} / t=f\left(Q_{t}\right)$ & $\begin{array}{c}Q_{e}=- \text { intercept } / \text { slope } \\
k_{2}=\text { slope }^{2} / \text { intercept } \\
h=\text { intercept }\end{array}$ \\
\hline
\end{tabular}

Table 2. Pseudo-second order kinetic parameters obtained by using the linear methods $\left(Q_{t}\right.$ and $Q_{e}, \mathrm{mg}_{\mathrm{g}} \mathrm{g}^{-1} ; k_{2}$, g. $\left.\mathrm{mg}^{-1} \cdot \mathrm{min}^{-1} ; t, \mathrm{~min}\right)$.

\begin{tabular}{|c|c|c|c|c|c|c|c|c|c|c|c|c|}
\hline \multirow{2}{*}{ Biosorbent } & \multicolumn{3}{|c|}{ Model 1} & \multicolumn{3}{|c|}{ Model 2} & \multicolumn{3}{|c|}{ Model 3} & \multicolumn{3}{|c|}{ Model 4} \\
\hline & $k_{2}^{*}$ & $Q_{e}$ & $R^{2}$ & $k_{2}$ & $Q_{e}$ & $R^{2}$ & $k_{2}$ & $Q_{e}$ & $R^{2}$ & $k_{2}$ & $Q_{e}$ & $R^{2}$ \\
\hline $\begin{array}{c}\text { Rhizopus sp. } \\
\text { R-18 }\end{array}$ & -0.07 & 45.5 & 0.999 & 0.05 & 45.5 & 0.904 & 0.05 & 46.0 & 0.836 & 0.04 & 46.4 & 0.836 \\
\hline $\begin{array}{l}\text { Penicillium } \\
\text { candidum }\end{array}$ & 0.02 & 42.6 & 0.999 & 0.08 & 42.0 & 0.975 & 0.08 & 41.9 & 0.929 & 0.08 & 42.0 & 0.929 \\
\hline $\begin{array}{l}\text { Penicillium } \\
\text { chrysogenum }\end{array}$ & 0.008 & 40.0 & 0.999 & 0.03 & 37.9 & 0.953 & 0.03 & 38.2 & 0.896 & 0.02 & 38.6 & 0.896 \\
\hline
\end{tabular}

*statistic non-significant parameters on the significance level $\alpha=0.05$

For all biosorbents tested, the best fit was achieved by Model 1. This model is statistically significant with a coefficient of determination near one for all three biosorbents. But this model gives statistically non-significant regression coefficients (in this case it is the intercepts) at a significance level of $\alpha=0.05$ (statistics data are not shown). Because of this, the model is not appropriate for the description of the experimental data. In the table 2 we can see that model 2 gives the best fits for all three biosorbents. It was observed that the kinetic parameters obtained from the three linear forms of pseudo-second order expressions were (models 2-4) very similar. From the aforementioned we can conclude that for description of kinetics of the sorption of methylene blue by the biomass of the fungi all three linear equations of the pseudosecond order can be used. 


\subsection{Non-linear regression analysis}

In the present study we used reaction based kinetic models, namely Lagergren pseudo-first order kinetic model, pseudo-second order model and pseudo- $n$th order kinetic model (Table 3). The kinetic parameters were determined using non-linear regression analysis by using Gauss-Newton algorithm. Table 4 shows obtained the predicted kinetic parameters. From the comparison of $A I C$ and $R^{2}$ it is clear that the pseudo- $n$th order kinetic model gives the best fit for all biosorbents.

Table 3. Kinetic models employed in this paper and their differential and non-linear equation forms.

\begin{tabular}{|c|c|c|c|}
\hline Kinetic model & Differential equation & Non-linear equation & Reference \\
\hline $\begin{array}{l}\text { Pseudo-first } \\
\text { order }\end{array}$ & $\frac{d Q_{t}}{d t}=k_{1}\left(Q_{e}-Q_{t}\right)$ & $Q_{t}=Q_{e}\left(1-e^{-k_{1} t}\right)$ & $\begin{array}{l}\text { LAGERGREN, } \\
1898\end{array}$ \\
\hline $\begin{array}{l}\text { Pseudo-second } \\
\text { order }\end{array}$ & $\frac{d Q_{t}}{d t}=k_{2}\left(Q_{e}-Q_{t}\right)^{2}$ & $Q_{t}=\frac{Q_{e}^{2} k_{2} t}{1+k_{2} t Q_{e}}$ & $\begin{array}{l}\text { HO, MCKAY, } \\
1998\end{array}$ \\
\hline $\begin{array}{l}\text { Pseudo- } n \text {th } \\
\quad \text { order }\end{array}$ & $\frac{d Q_{t}}{d t}=k_{n}\left(Q_{e}-Q_{t}\right)^{n}$ & $Q_{t}=Q_{e}-\left[Q_{e}^{1-n}-(1-n) k_{n} t\right]^{1 /(1-n)}$ & $\begin{array}{l}\text { ÖZER, } \\
2007\end{array}$ \\
\hline
\end{tabular}

Table 4. Kinetic model parameters obtained by using non-linear regression analysis (1-Rhizopus sp. R-18; 2$P$. candidum; 3-P. chrysogenum; $k_{1}, k_{2}$ and $k_{n}$ are rate constants; $n$ is the order of reaction; $Q_{e}$ is the amount of dye adsorbed onto biosorbent at equilibrium $\left(\mathrm{mg} \cdot \mathrm{g}^{-1}\right)$.

\begin{tabular}{|c|c|c|c|c|c|c|c|c|c|c|c|c|c|}
\hline \multirow{2}{*}{$\begin{array}{l}\text { Bio- } \\
\text { sorbent }\end{array}$} & \multicolumn{4}{|c|}{ Pseudo-first order kinetic } & \multicolumn{4}{|c|}{ Pseudo-second order kinetic } & \multicolumn{5}{|c|}{ Pseudo- $n$th order kinetic } \\
\hline & $k_{1}$ & $Q_{e}$ & $R^{2}$ & $A I C$ & $k_{2}$ & $Q_{e}$ & $R^{2}$ & $A I C$ & $k_{n}$ & $Q_{e}$ & $n$ & $R^{2}$ & $A I C$ \\
\hline 1. & 1.7 & 45.2 & 0.962 & 26.1 & 0.05 & 46.1 & 0.984 & 15.4 & 0.001 & 48.0 & 3.1 & 0.991 & 11.0 \\
\hline 2. & 1.6 & 41.1 & 0.998 & 10.0 & 0.08 & 41.9 & 0.996 & -4.9 & 0.004 & 43.1 & 3.0 & 0.999 & -22.0 \\
\hline 3. & 0.5 & 37.2 & 0.927 & 111 & 0.02 & 38.7 & 0.981 & 14.3 & $5.10^{-7}$ & 43.0 & 3.8 & 0.997 & -2.31 \\
\hline
\end{tabular}
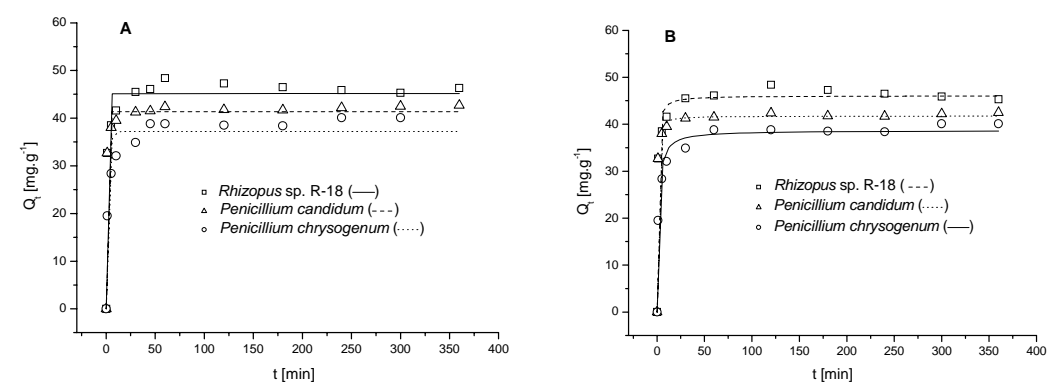

Fig. 1. Pseudo-first (A) and pseudo-second (B) order kinetics by non-linear method by using Gauss-Newton algorithm and experimental kinetics for the sorption of methylene blue onto three different biosorbents.

The order of adsorption reaction ( $n$ ) was found to be between 3.0 and 3.8. From the aforementioned we can conclude that the adsorption of methylene blue by the biomass of the fungi is governed by a reaction order higher than 2. But the model gives 
statistically insignificant coefficients (in the case it is $k_{n}$ ) for all three biosorbents (statistics data are not shown). This excludes the use of this model for the description of our experimental data. We can't forget that this model, contrary to the two others, is a three parameter model. On the one hand the higher number of parameters gives us a better fit, but on the other hand the statistical significance of the regression coefficients is lower. One possible way to increase the statistical significance of the regression coefficients is to increase the number of experimental measurements. Because of this, in this work, the pseudo-second order kinetic model seems to be the best fitting for all three biosorbents (Fig. 1).

\subsection{Comparison of linear and non-linear regression analysis}

To compare model fits between the different linearizations and the nonlinear pseudo-second order kinetic model equation, the best-fit lines for each linearization were transformed back to sorbed concentrations $\left(Q_{t}\right)$ and error analysis as well as $A I C$ were using for comparison goodness-of-fit measure between the different linearizations and the non-linear equation (Table 5).

Table 5. Statistic parameters for the nonlinear and linearized pseudo-second order kinetic equations for three different biosorbents.

\begin{tabular}{cccccc}
\hline \multirow{2}{*}{ Biosorbent } & $\begin{array}{c}\text { Statistic* } \\
\text { parameter }\end{array}$ & Model 2 & Model 3 & Model 4 & $\begin{array}{c}\text { Nonlinear } \\
\text { model }\end{array}$ \\
\cline { 3 - 5 } & SSE & 32.4 & 31.8 & 31.8 & 30.9 \\
Rhizopus & SAE & 13.6 & 13.4 & 13.9 & 13.8 \\
sp. R-18 & red $\chi^{2}$ & 0.086 & 0.084 & 0.089 & 0.084 \\
& AIC & $\mathbf{2 0 . 9}$ & $\mathbf{2 0 . 7}$ & $\mathbf{2 0 . 7}$ & $\mathbf{2 0 . 4}$ \\
& SSE & 5.71 & 5.49 & 5.71 & 5.72 \\
Penicillium & SAE & 6.1 & 5.90 & 6.10 & 6.23 \\
candidum & red $\chi^{2}$ & 0.016 & 0.015 & 0.016 & 0.016 \\
& AIC & $\mathbf{0 . 0 8 8}$ & $\mathbf{- 0 . 3 8}$ & $\mathbf{0 . 0 8 8}$ & $\mathbf{0 . 1 1 5}$ \\
& SSE & 42.5 & 42.4 & 28.4 & 28.1 \\
Penicillium & SAE & 18.2 & 17.6 & 14.2 & 14.2 \\
chrysogenum & red $\chi^{2}$ & 0.160 & 0.165 & 0.126 & 0.124 \\
& AIC & $\mathbf{2 4 . 3}$ & $\mathbf{2 4 . 3}$ & $\mathbf{1 9 . 9}$ & $\mathbf{1 9 . 8}$
\end{tabular}

"SSE- the sum of the squares of the errors; SAE- the sum of the absolute errors; red $\chi^{2}$ - reduced Chi-square analysis; AIC- Akaike's information criterion .

From the statistical analysis we can conclude, that the linearized models 2, 3 and 4 give us very similar fits to the non-linear models. The best example for this is biosorbent $P$. candidum with an $A I C$ value of -0.38 (Model 3). This fact can be described in the following way: the linear regression analysis was done using the leastsquares methods. In order to use this method some conditions known from statistics must be met. If we want to use the linear forms of the kinetic equations of the pseudo- 
second order the data from the measurement must be transformed. The transformation probably changed the conditions in a positive way, and that caused that the linear models (except model 1) give very similar or better fits than the non-linear models (look at $P$. candidum in the Table 5). As mentioned in the literature this doesn't always have to be the case the data transformation can also negatively affect the estimate of the regressive model. Therefore, the primary drawback to linearization is not the inability to provide similar parameter estimates as the nonlinear equation but rather the inability to provide poor (or good) model fits when the data don't (or do) conform to the kinetic model.

\section{Conclusions}

The sorption of methylene blue by three fungal biosorbents was found to be rapid whereby the equilibrium was reached during one hour. The results confirmed the applicability several kinetic models, namely pseudo-second and pseudo $n$th order kinetic models. Present investigation further showed that the search for best-fit kinetic model using linearization technique is not an appropriate technique to predict biosorption kinetics. The non-linear methods would be more appropriate techniques in predicting the biosorption kinetics.

\section{References}

AKSU, Z.: Application of biosorption for the removal of organic pollutants: a review. Process. Biochem., 40, 2005, 997-1026.

AL-DEGS, Y. S., EL-BARGHOUTHI, M. I., ISSA, A. A., KHRAISHEH, M. A., WALKER, G. M.: Sorption of $\mathrm{Zn}(\mathrm{II}), \mathrm{Pb}(\mathrm{II})$ and $\mathrm{Co}(\mathrm{II})$ using natural sorbents: equilibrium and kinetic studies. Water Res., 40, 2006, 2645-2658.

BOLSTER, H. C., HORNBERGER, M. G.: On the use of linearized Langmuir equations. Soil Sci. Soc. Am. J., 71, 2007, 796-1806.

HO, Y. S., MCKAY, G.: Kinetic models for the sorption of dye from aqueous solution by wood. Trans. Inst. Chem. Eng., 76, 1998, 183-191.

HO, Y. S., NG, J. C. Y., MCKAY, G.: Kinetics of pollutant sorption by biosorbent: review. Sep. Pur. Methods, 29, 2000, 189-232.

KUMAR, K. V.: Linear and non-linear regression analysis for the sorption kinetics of methylene blue onto activated carbon. J. Hazard. Mater., 137, 2006, 1538-1544.

LAGERGREN, S.: About the theory of so-called adsorption of soluble substances. K. Sven. Vetenskapsakad. Handl., 24, 1898, 1-39.

ÖZER, A.: Removal of $\mathrm{Pb}(\mathrm{II})$ ions from aqueous solutions by sulphuric acid-treated wheat bran. J. Hazard. Mater., 141, 2007, 753-761.

SVILOVIĆ, S., STIPIŠIĆ, D. R.: Modeling batch kinetics of copper ions sorption using synthetic zeolite NaX. J. Hazard. Mater., 2009 (in press). 